
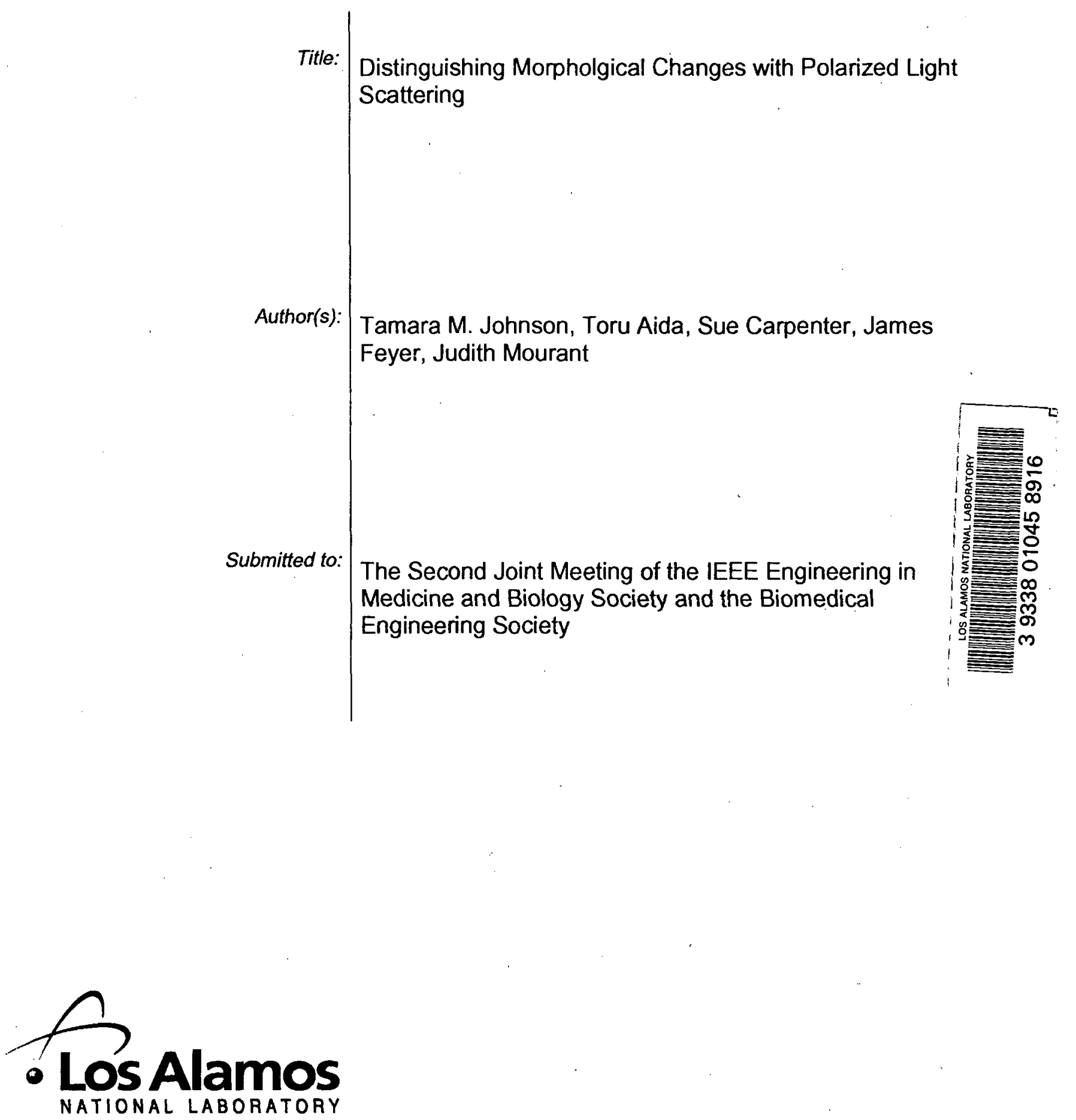

Los Alamos National Laboratory, an affirmative action/equal opportunity employer, is operated by the University of California for the U.S Department of Energy under contract W-7405-ENG-36. By acceptance of this article, the publisher $=$ gnizes that the U.S. Government retains a nonexclusive, royalty-free license to publish or reproduce the published form of this contr $\equiv \mathrm{n}$, or to allow others to do so, for U.S Government purposes. Los Alamos National Laboratory requests that the publisher identify this articu as work performed under the auspices of the U.S. Department of Energy. Los Alamos National Laboratory strongly supports academic freedom and a researcher's right to publish; as an institution, however, the Laboratory does not endorse the viewpoint of a publication or guarantee its technical correctness. 


\title{
DISTINGUISHING MORPHOLOGICAL CHANGES WITH POLARIZED LIGHT SCATTERING
}

\author{
Judith R. Mourant, Tamara M. Johnson, Toru Aida, Sue Carpenter and James P. Freyer \\ Bioscience Division, MS E535, Los Alamos National Laboratory,
} Los Alamos NM, 87545

\begin{abstract}
Results of work determining how different biological structures contribute to light scattering will be presented. Further, measurements of phantoms that mimic structural changes expected in vivo will be presented. It is found that polarized measurements can discriminate between phantoms with similar properties. Keywords - biomedical optics, noninvasive measurements, light scattering, cancer detection
\end{abstract}

\section{INTRODUCTION}

Non-invasive optical diagnostics have the potential to diagnose pre-cancerous conditions. Most cancers arise in epithelial tissues which are composed almost entirely of cells. The work described here builds on our early work to understand what structures in biological cells scatter light (1). There will be three parts to this talk; 1 . A study of what size structures contribute to reflectance from epithelial tissues. 2. A study of how light scattering changes with the rate at which cells are growing. 3. The development of phantoms to mimic the changes found in 2 and testing of a endoscopically compatible measurement system to distinguish morphological changes such as those found in 2 .

\section{METHODS}

Angular-depenedent light scattering measurements

- Angular-dependent, polarized, light scattering measurements are made. Briefly, a dilute suspension of cells is placed in a sample holder that is illuminated with a polarized $\mathrm{HeNe}$ laser beam. Light scatter from the cells is detected with an avalanche photodiode that can be rotated around the sample. Both the polarization of the incident and detected light can be changed.

\section{Biological samples}

The samples we measure are carcinogenic rat prostate epithelial cells. They are harvested for measurement in either the plateau or the exponential phases of growth. For measurement they are kept in ice-cold saline.

\section{Monte Carlo simulations}

The propagation of light through turbid media such as tissue is modeled using Monte Carlo simulations. The simulation can incorporate elliptical as well as spherical scatterers. It also allows for counting of the number of times the collected photons scattered off of particles of a particular class.

\section{Tissue Phantoms}

Tissue phantoms were made using gelatin, polystyrene spheres and intralipid. The gelatin is used in order make a solid phantom, the polystyrene spheres allow for easy alterations to the phantoms by using different size spheres, and the intralipid is used so that the phantoms have some cross-polarized light scattering intensity (1).

\section{Reflectance/Elastic scatter measurements}

A fiber optic probe has been developed that allows for measurement of backscattered light with different polarizations (2). A schematic of the distal end of the probe is shown in Fig. 1. There is one light delivery fiber and four light collection fibers. The lines over the fibers represent the polarization of the light. As described in reference 2 , the measurements with this probe at a single wavelength are independently sensitive to both the size and concentration of scattering structures. In this work wavelength dependent measurements are made. The light source is a tungsten lamp. Detection is performed with a TE-cooled CCD.

\section{RESULTS}

Light scattering occurs primarily from small structures with dimensions on the order of 10's of microns, and from larger structures such as mitochondria and nucleoli. Based on experimental knowledge of light scattering from different size structures as a function of angles, Monte Carlo simulations can be used to determine how different structures contribute to the reflectance/elastic scatter signal in different geometries. A common geometry for reflectance/elastic scatter measurements is two optical fibers in close proximity on the surface of a tissue. One of the fibers delivers light to the tissue while the other fiber collects light. We have found that as the separation between the two fibers increases the contribution of the smallest scatterers to the light scattering signal decreases. Further, we have found that in most geometries particles of the size of the mitochondria and nucleoli are the major contributors to light scattering.

Light scattering measurements of cells in the exponential and plateau phases of growth demonstrate that there are significant changes. Ratios of scattering from exponential and plateau phase epithelial and fibroblast cells are shown. in Fig. 1. The results clearly depend on cell type. For the fibroblast cells, more scattering occurs from the cells in the exponential phase cells. For the epithelial cells, there is more scattering from the plateau phase cells at some 
angles. Furthermore, the angular dependence varies for the epithelial cells.

We have developed tissue phantoms to mimic the small light scattering changes we observed between plateau and exponential phase cells. These phantoms were measured with a fiber optic probe that is sensitive to light polarization. Fig. 3 demonstrates that we are able to differentiate the phantoms called mix 11 and mix 12. The left axis is the ratio of light intensity at $650 \mathrm{~nm}$ collected by fibers 1 and 4 in the probe shown in Fig. 1. The bottom axis is the ratio of the light intensity $\mathrm{t} 650 \mathrm{~nm}$ collected by fibers 1 and 3 . Fig. 4 shows the angular dependence of light scattered from dilute versions of the phantoms. The results in Fig. 4 are for light polarized parallel to the scattering plane. Comparison with results in reference 2 shows that the scattering is similar to that from epithelial cells.

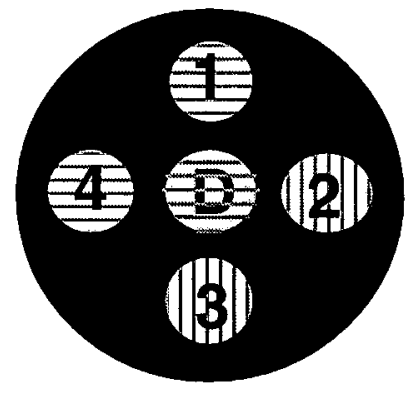

Fig. 1. Schematic of the polarization probe.

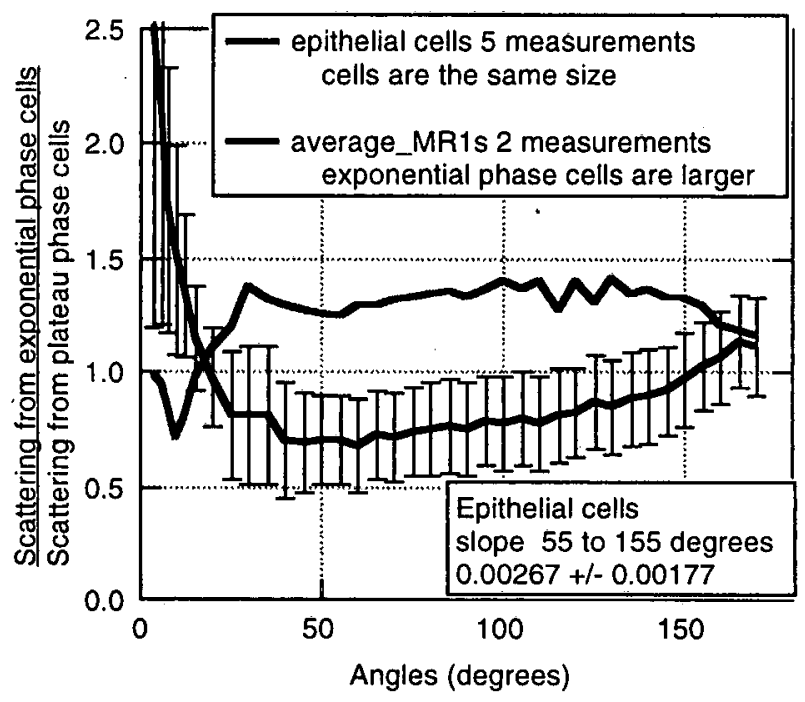

Fig. 2 Ratio of light scattering from epithelial cells harvested in the exponential phase of growth to the light scattering from epithelial cells harvested in the plateau phase of growth.

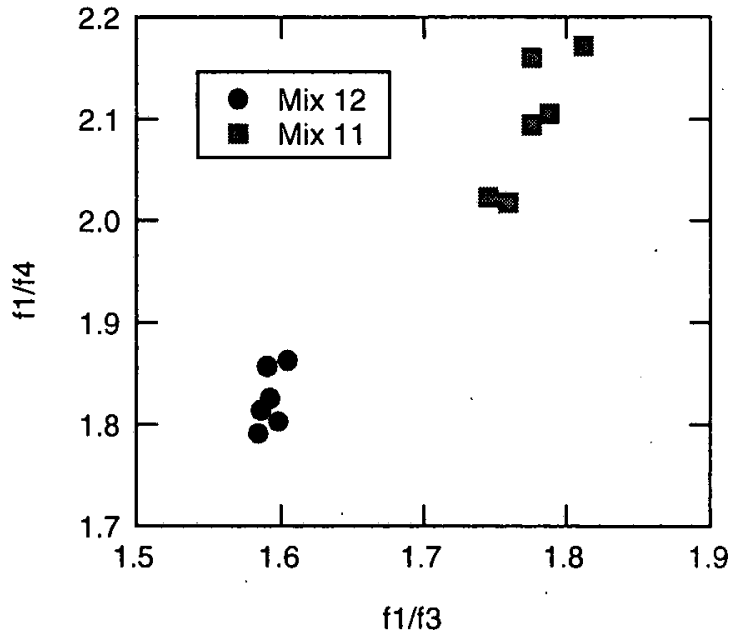

Fig. 3. Results of elastic scatter measurements of phantoms that mimic dense suspensions of biological cells.

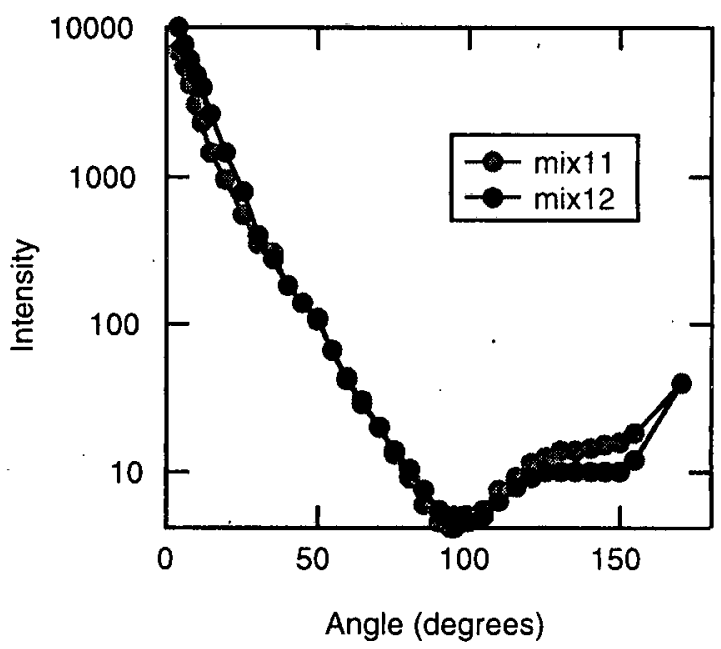

Fig. 4. Angular dependence of light scattered from dilute versions of the phantoms. The results in are for light polarized parallel to the scattering plane.

\section{References}

1. J. R. Mourant, T. M. Johnson, S. Carpenter, A. Guerra, T. Aida, and J. P. Freyer, "Polarized angular-dependent spectroscopy of epithelial cells and epithelial cell nuclei to determine the size scale of scattering structures," in press Journal of Biomedical Optics.

2. J. R. Mourant, T M. Johnson, J. P. Freyer, "Characterizing mammalian cells and cell phantoms with polarized fiber-optic backscattering measurements," Appl. Opt. 40: 5114 - 5123 (2001). 


\title{
Pathogen Detection Program Review 10/7/02
}

\author{
Judith R. Mourant \\ Bioscience Division
}

Los Alamos National Laboratory 


\section{Outline}

- Review data

- Visual examination of the data

- SIMCA analysis

- Review of SIMCA method

- Application to this data

- Choice of measurement wavelengths

- Conclusions 


\section{Summary of data since last program review}

- B. Globigii spores on tape (different concentrations)

- Computer dust with and w/o BG

- B. Cereus spores measured at Edgewood

- BC or BG or on tape

- Chair dust with and w/o BG, BC or BP 


\section{Excitation - Emission Images}

Excitation: $200-600 \mathrm{~nm}$

Emission: 210 - $700 \mathrm{~nm}$

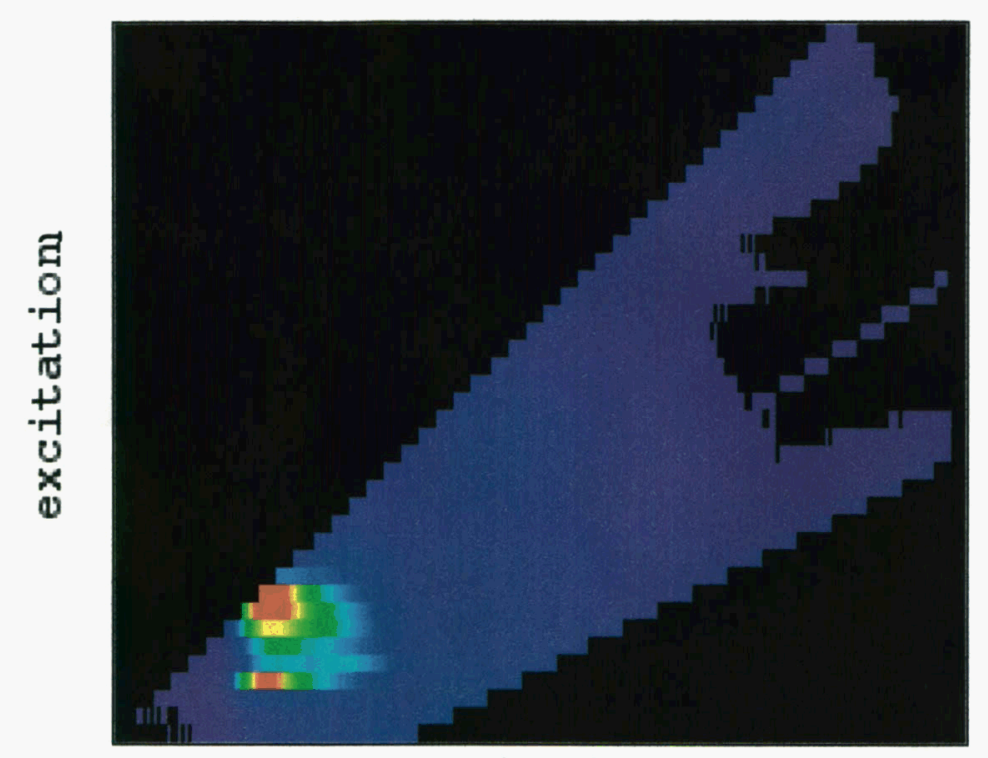

emission

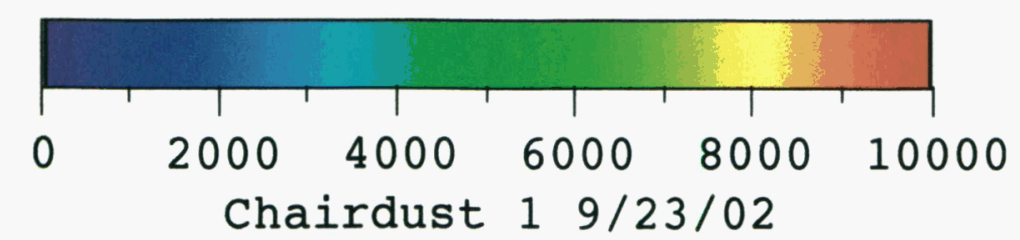

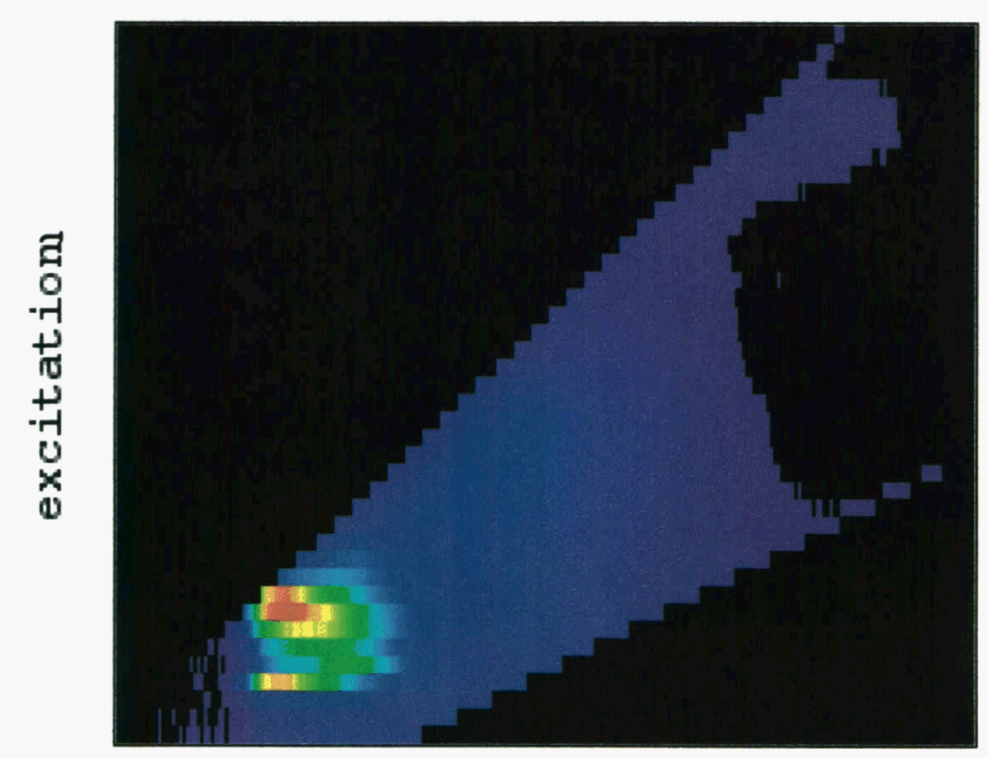

emission

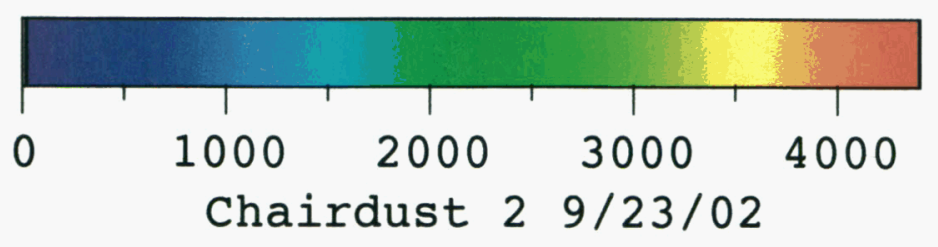



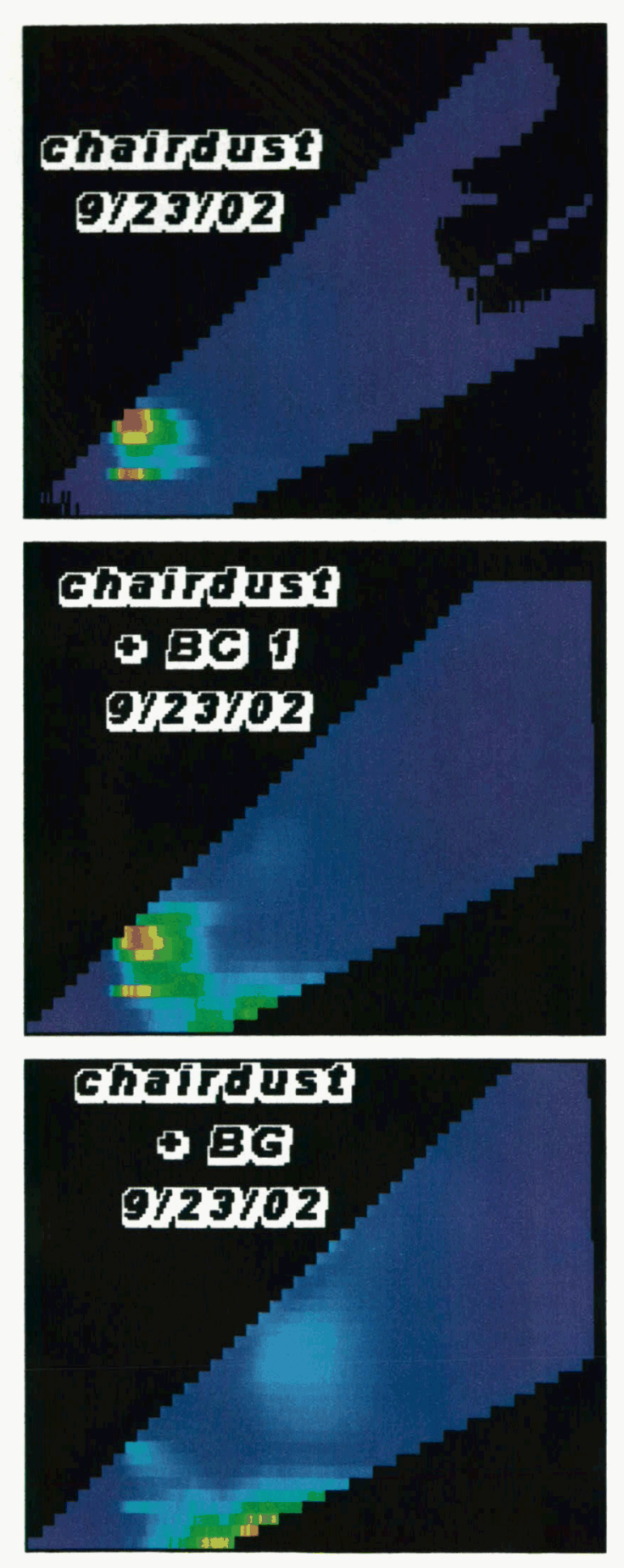
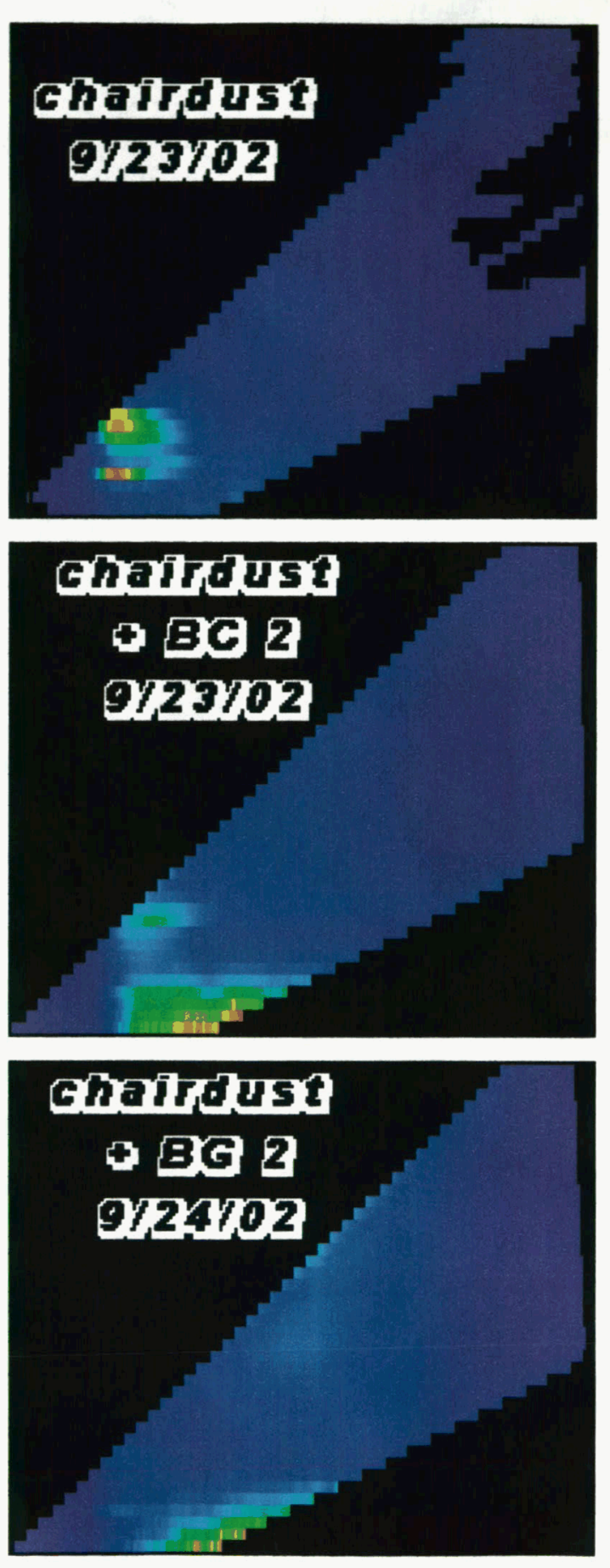
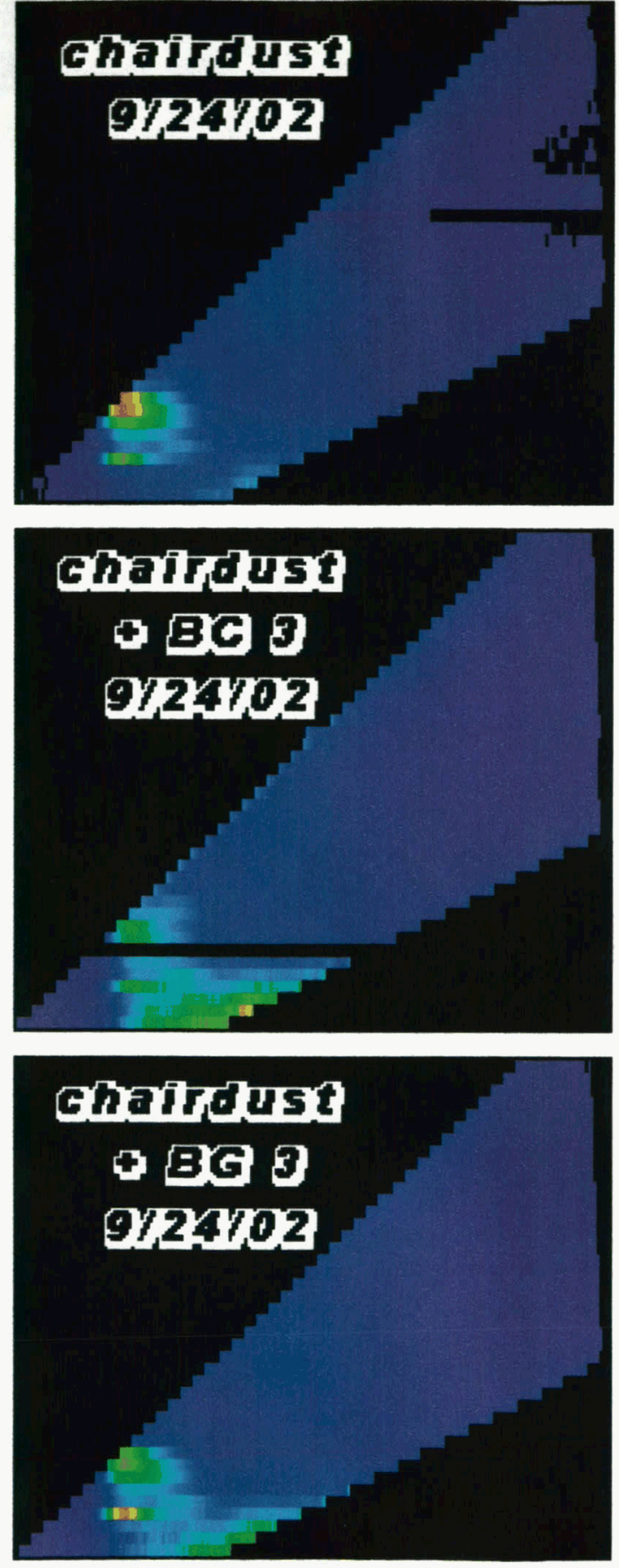

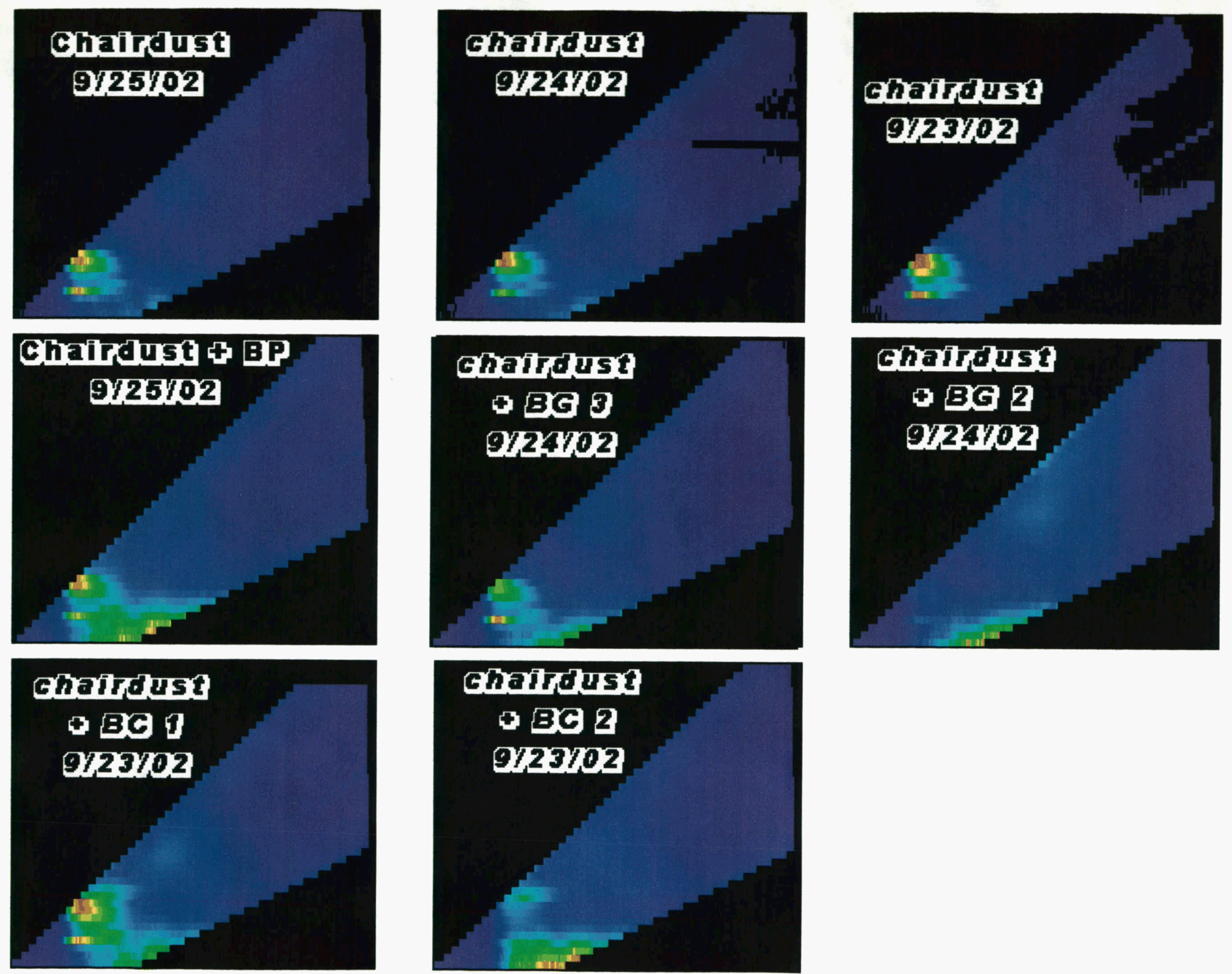


\section{Observations}

- Bacillus species

- Decreases the apparent fluorescence from the tape

- Causes an increased fluorescence at "long wavelengths" with short wavelength excitation

- This feature is enhanced by amplification by the spectrometer

- Sometimes fluorescence is apparent due to longer wavelength emission 


\section{Concentration study}
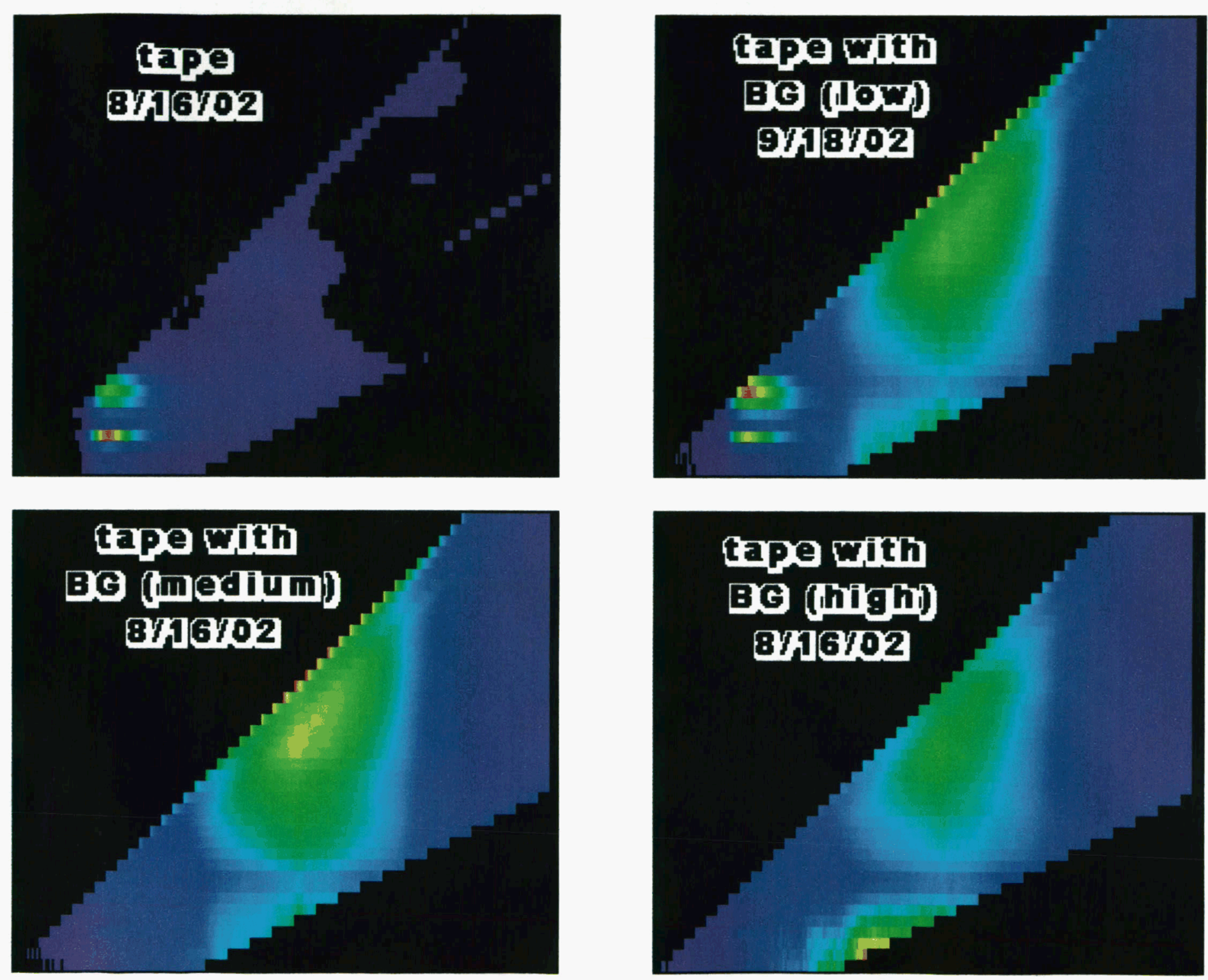


\section{Observations}

- The chair dust has very little fluorescence

- Spectra are similar to that of tape

- At low concentration the BG does not mask the tape fluorescence 


\section{Comparison of $\mathrm{BG}$ and $\mathrm{BC}$}
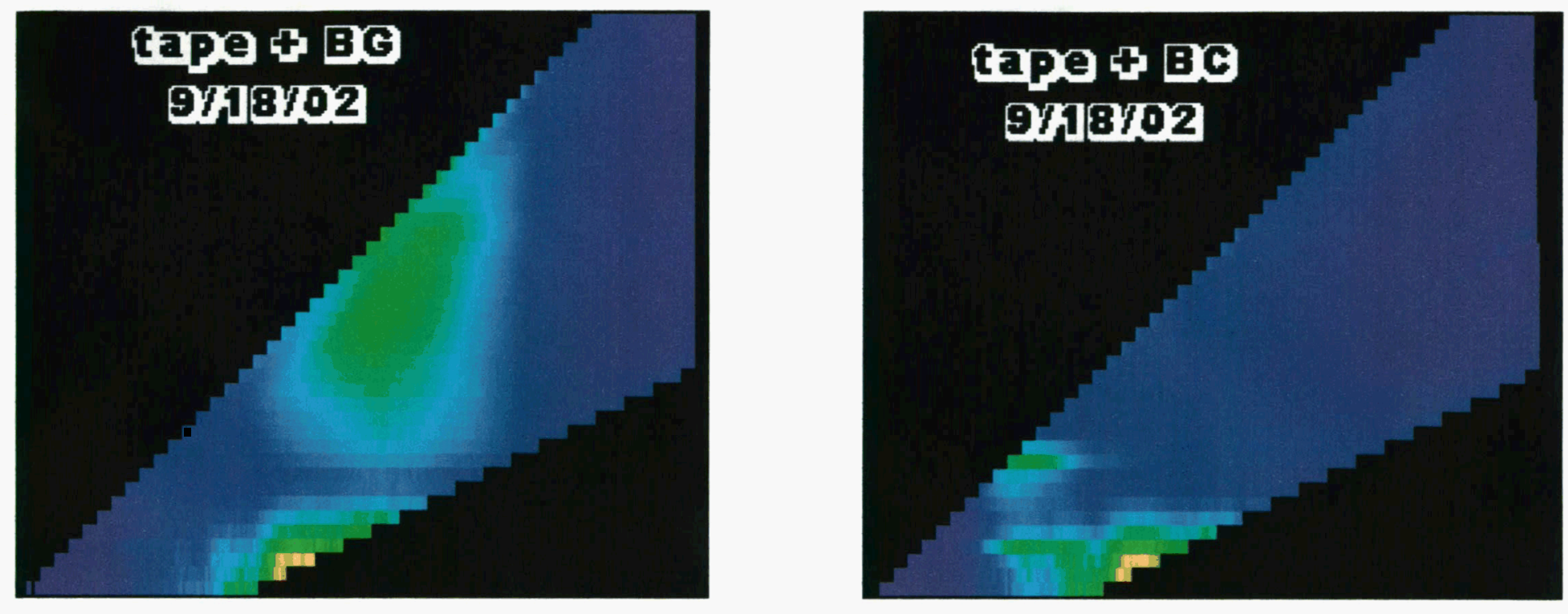

Need a concentration study of BC 


\section{Computer dust is very different from chair dust.}
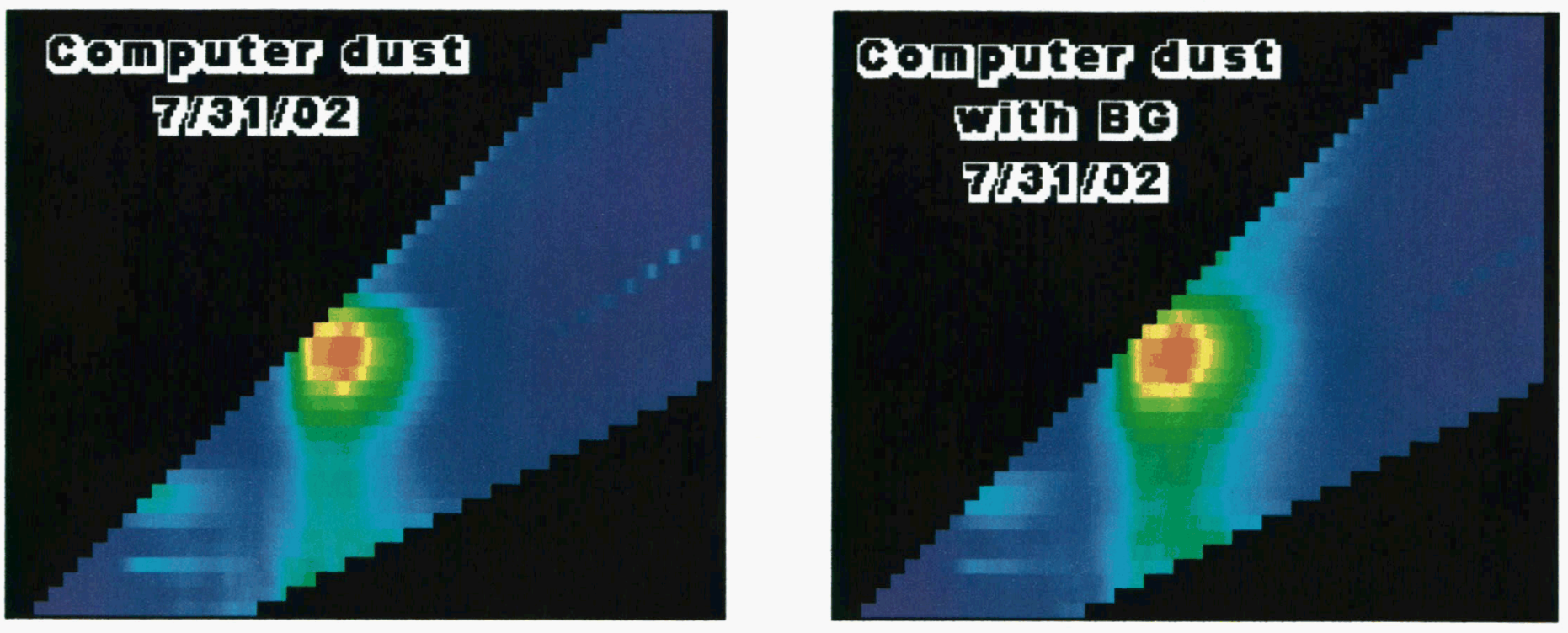


\section{SIMCA: Soft independent modeling of class analogy}

- Creates principle component models for each training set

- Modeling power can be used to chose variables

- Prediction possibilities

- Sample only fits one pre-defined category

- Sample doesn't fit any pre-defined category

- Sample fits into more than one pre-defined category; ranks choices 


\section{SIMCA implementation}

- 4 groups

- Group 1: 4 Chair dust samples

- Collected on 3 different days

- Group 2: 4 BC samples

- 3 with dust, 1 on tape only

- Collected on 3 different days

- Group 3: 4 BG samples

- 3 with dust, 1 on tape only

- Collected on 3 different days

- Group 4: PB samples

- 2 with chair dust, 2 on tape

- All collected on the same day. 


\section{SIMCA implementation}

- Run 1

- All emission spectra were concatenated

- The data were normalized to have the same integrated values of emission between 389 and $395 \mathrm{~nm}$ due to $360 \mathrm{~nm}$ excitation

- Cut out most but not all of the reflection 


\section{Example of Data}

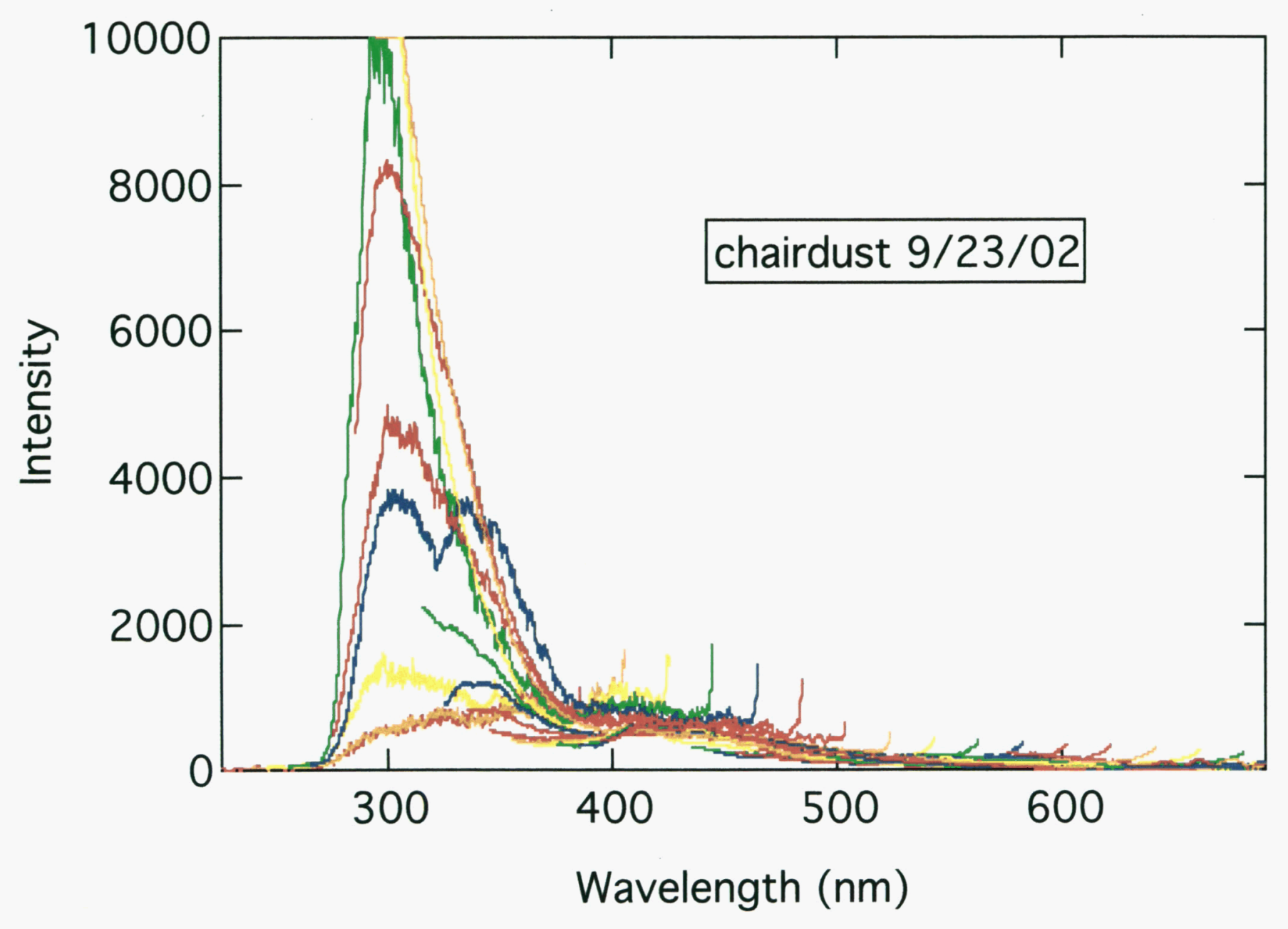




\section{Example of Concatenated Spectra}

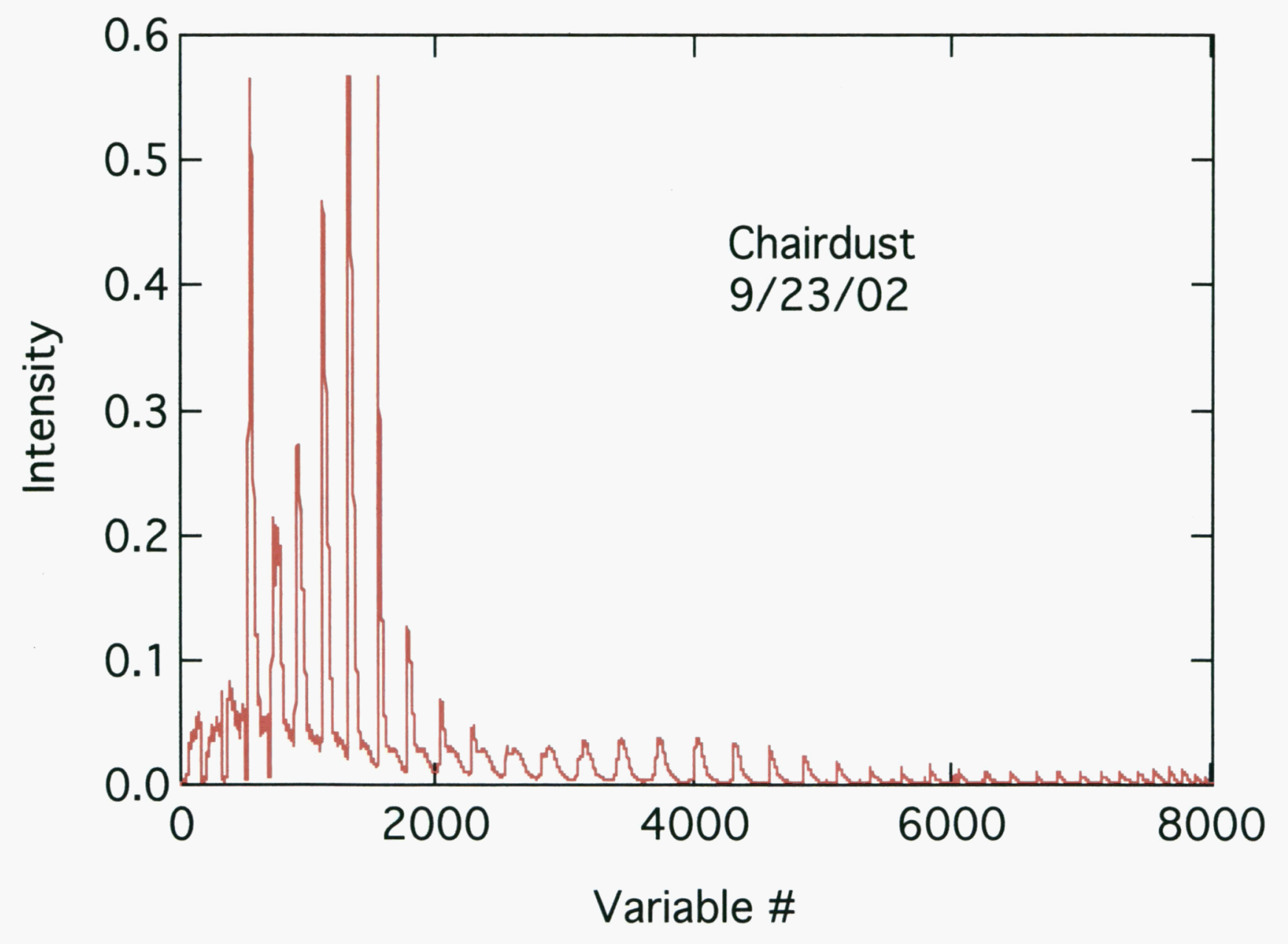




\section{SIMCA Results}

- Developed a model based on a principal component analysis of each group

- Each spectra was properly assigned to the correct group

- Used three principle components for each group 


\section{SIMCA Results cont.}

- Demonstrated that SIMCA can not make a model out of any arbitrary data

- Took each of the 16 spectra and "randomly" assigned each one to one of four groups

- 2 spectra were not placed in the assigned group.

- Assigned all chair dust spectra to one group, randomly assigned the other 12 spectra to 1 of 3 groups

- 1 spectra was not placed in the assigned group 


\section{Modeling Power}

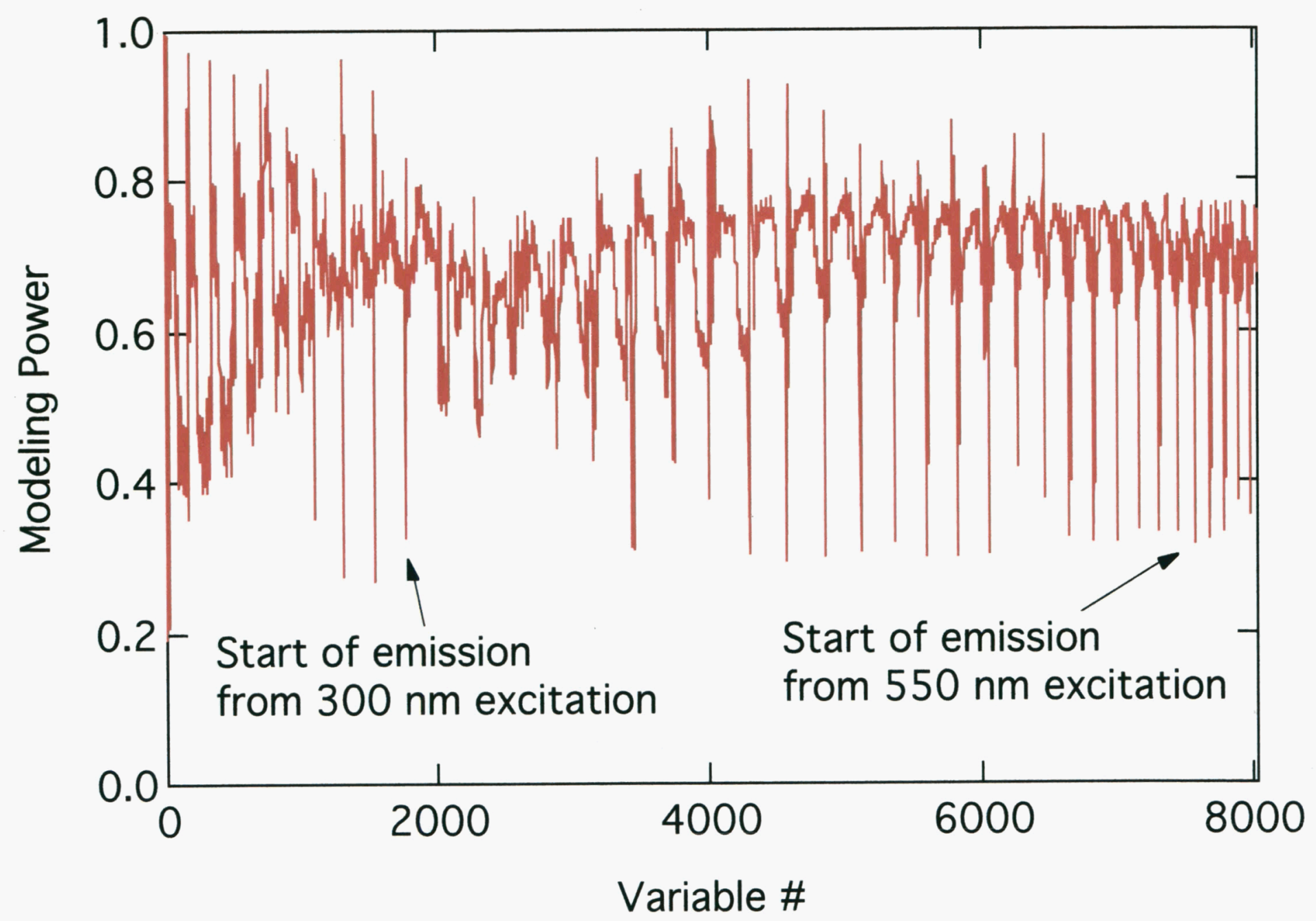




\section{Cutting out variables}

- Run 2

- Cut out all data below $300 \mathrm{~nm}$ excitation

- Run 3

- Cut out all data above $550 \mathrm{~nm}$ excitation

- For both runs all spectra were placed in the correct group 


\section{Discussion/Future}

- The proper way to perform SIMCA is on a training data set and then a testing data set.

- Need more data

- Different types of dust

- Different concentrations of BG, BP, BC and other relevant spores/bacteria

- All data needs to be taken under the same conditions 


\section{Discussion/Future}

- May be able to exclude wavelengths below $400 \mathrm{~nm}$

- Preference is to continue obtaining data over the largest possible range

- Possibly the reason cutting out some of the data did not affect the SIMCA results is that there is redundant information at this point

- May need this information when we look all the bacteria with all different backgrounds.

- Prefer less reflection 